\title{
Electroweakly interacting Dirac dark matter
}

\author{
Natsumi Nagata ${ }^{1,2, *}$ and Satoshi Shirai ${ }^{3, \dagger}$ \\ ${ }^{1}$ William I. Fine Theoretical Physics Institute, School of Physics and Astronomy, \\ University of Minnesota, Minneapolis, Minnesota 55455, USA \\ ${ }^{2}$ Kavli Institute for the Physics and Mathematics of the Universe (WPI) Todai Institutes \\ for Advanced Study, University of Tokyo, Kashiwa 277-8583, Japan \\ ${ }^{3}$ Deutsches Elektronen-Synchrotron (DESY), 22607 Hamburg, Germany
}

(Received 14 November 2014; published 30 March 2015)

\begin{abstract}
We consider a class of fermionic dark matter candidates that are charged under both the $\mathrm{SU}(2)_{L}$ and $\mathrm{U}(1)_{Y}$ gauge interactions. In this case a certain amount of dark matter-Higgs couplings, which can split the dark matter into a pair of Majorana fermions, should be present to evade the constraints from the dark matter direct detection experiments. These effects may be probed by means of the dark matter-nucleus scattering via the Higgs-boson exchange process, as well as the electric dipole moments induced by the dark matter and its $\mathrm{SU}(2)_{L}$ partner fields. In this article, we evaluate them with an effective field approach. It turns out that the constraints coming from the experiments for the quantities have already restricted the dark matter with hypercharge $Y \geq 3 / 2$. Future experiments have sensitivities to probe this class of dark matter candidates, and may disfavor the $Y \geq 1$ cases if no signal is observed. In this case, only the $Y=0$ and $1 / 2$ cases may be the remaining possibilities for the $\mathrm{SU}(2)_{L}$ charged fermionic dark matter candidates.
\end{abstract}

DOI: 10.1103/PhysRevD.91.055035

PACS numbers: 12.60.-i, 95.35.+d

\section{INTRODUCTION}

Weakly interacting massive particles (WIMPs) are wellknown candidates for dark matter (DM) in our Universe. The thermal relic abundance of a TeV-scale WIMP can be consistent with the observed DM density, $\Omega_{\mathrm{DM}} h^{2}=$ $0.1196 \pm 0.0031$ (68\% C.L.) [1]. The WIMP DM is required to be electrically and color neutral; however, its electroweak (EW) charges are still scarcely constrained. The EW charges are characterized by the number of $\mathrm{SU}(2)_{L}$ components $n$ and its $\mathrm{U}(1)_{Y}$ hypercharge $Y$. Various DM candidates with different combinations of $(n, Y)$ have been discussed so far in the literature [2]. For instance, an $n=5$ fermion or an $n=7$ scalar multiplet with $Y=0$ is often stressed as "minimal DM" for its automatic DM stabilization mechanism [3]. A wino $(n=3$ and $Y=0)$ is also a good DM candidate in the supersymmetric Standard Model. Moreover, a remnant discrete symmetry resulting from the grand unified symmetry may give rise to stable DM candidates charged under the $\mathrm{SU}(2)_{L} \otimes \mathrm{U}(1)_{Y}$ gauge interactions [4].

In this article, we especially focus on DM with $Y \neq 0$. These multiplets distinguish themselves from others as they can have vectorlike mass terms. In fact, such possibilities have been already excluded by the DM direct detection experiments if the DM has only the gauge interactions; the $Z$-boson exchanging processes induce the vector-vector coupling between the DM and quarks, which gives too large DM-nucleus elastic scattering cross sections.

\footnotetext{
*natsumi.nagata@ipmu.jp

†satoshi.shirai@desy.de
}

However, small couplings between the Higgs field and the DM, even via higher-dimensional operators whose cutoff scale $\Lambda$ is much higher than the DM mass, can allow the scenario to evade the constraint. This is because a nonzero Higgs vacuum expectation value makes the Dirac fermion (complex scalar) split into two Majorana fermions (real scalars) DM and $\mathrm{DM}^{\prime}$. In this case, the DM cannot interact with quarks via the vector-vector interaction and thus the DM-nucleon elastic scattering cross sections are small enough to avoid the experimental constraints. Therefore, a model with hypercharged DM should involve some mechanism to generate couplings between the Higgs field and the DM. One of the most famous examples for such DM is a Higgsino-like $(n=2$ and $Y=1 / 2)$ neutralino in the supersymmetric Standard Model. Other models are discussed in Refs. [4,5]. This kind of model also predicts some model-dependent physical consequences which are not determined only with the EW charges of the DM.

The goal of this article is to extract the physical consequences of the hypercharged DM with coupling to the Higgs boson, in model-independent manners. Too tiny DM-Higgs coupling reduces the mass splitting between the $\mathrm{DM}$ and $\mathrm{DM}^{\prime}, \Delta m$, and the inelastic scattering $\mathrm{DM}+N \rightarrow$ $\mathrm{DM}^{\prime}+N$ again gives a strong constraint. To avoid it, there is a lower bound on the Higgs and DM couplings. The coupling can also induce the electric dipole moments (EDMs) of quarks and leptons, as well as the scalar-type DM scattering with nucleus, which will be probed by present and future progressing experiments. We mainly focus on the case of fermionic DM, and show that future experiments have sensitivities to probe this class of DM 
candidates and may disfavor the $Y \geq 1$ cases if no signal is observed. At the end of this article, we briefly comment on the scalar DM.

\section{DARK MATTER AND HIGGS COUPLINGS}

Let $\psi_{m}$ be the $\mathrm{SU}(2)_{L} n$-tuplet Dirac fermions with hypercharge $Y>0$. The index $m$ labels the eigenvalues of $T_{3}$ with $T_{a}(a=1,2,3)$ the $n$-dimensional representation of the generators of the $\mathrm{SU}(2)_{L}$ gauge group. In the basis, $T_{ \pm} \equiv T_{1} \pm i T_{2}$ and $T_{3}$ are represented by $\left(T_{ \pm}\right)_{l m}=$ $\sqrt{(j \mp m)(j \pm m+1)} \delta_{l, m \pm 1}$ and $\left(T_{3}\right)_{l m}=m \delta_{l m}$ with $j \equiv$ $\frac{n-1}{2}$. We require that the multiplets should contain the neutral components; the condition reads $Y \leq j$ and $(j-Y)$ being an integer. Further, the lightest neutral component is assumed to be the dominant component of DM in the Universe. The mass term of the multiplets is given by

$$
\mathcal{L}_{\text {mass }}=-\mu \bar{\psi} \psi,
$$

with $\mu$ taken to be real and positive, without loss of generality. We assume it to be around the $\mathrm{TeV}$ scale in the following discussion. Without UV-physics effects, the fermions interact with the Standard Model sector only through the gauge interactions. As discussed in the Introduction, however, it is required to include the effects to evade the constraints coming from the DM direct detection experiments. ${ }^{1}$ Such effects are described by the following effective operators that break the conservation of the fermion number associated with the multiplets ${ }^{2}$ :

$$
\begin{aligned}
\mathcal{L}_{\text {eff }}^{(c)}= & \frac{c_{s}}{2 \Lambda^{(4 Y-1)}} \sum_{M, m, m^{\prime}}\left\langle j m j m^{\prime} \mid(2 Y) M\right\rangle\left[(H)_{M}^{4 Y}\right]^{*} \overline{\psi_{m}^{c}} \psi_{m^{\prime}} \\
& + \text { H.c., }
\end{aligned}
$$

where $c_{s}$ is an $\mathcal{O}(1)$ dimensionless constant and $\psi^{c}$ is the antiparticle field of $\psi ; \Lambda$ is taken to be real and positive without loss of generality. $H=\left(H^{+}, H^{0}\right)^{T}$ is the Higgs field, $(H)^{k}$ is composed of $k$ Higgs fields to form an isospin- $k / 2$ object and defined such that its lowest component is given by $\left(H^{0}\right)^{k}$, and $\left\langle j m j^{\prime} m^{\prime} \mid J M\right\rangle$ are the Clebsch-Gordan coefficients. Notice that from their symmetry properties, $\left\langle j m j m^{\prime} \mid(2 Y) M\right\rangle=(-1)^{2(j-Y)} \times$ $\left\langle j m^{\prime} j m \mid(2 Y) M\right\rangle$ follows, and thus the operators in Eq. (2) vanish unless $(j-Y)$ is an integer. The condition is, however, always satisfied in the present scenario since we have assumed that the multiplets have the neutral components.

In general, the UV-physics effects that induce the above operator also generate other operators that have lower mass

\footnotetext{
${ }^{1}$ The constraints can be also avoided if $\mu$ is much higher than the TeV scale. Such a possibility is studied in Ref. [6].

${ }^{2}$ In addition, there is a similar pseudoscalar operator. However, we find that it plays no role in the following discussion.
}

dimensions but give no contribution to the mass splitting between the neutral components. Among them, the following dimension-five operators give rise to the dominant contribution to the low-energy physics ${ }^{3}$ :

$$
\begin{aligned}
\mathcal{L}_{\text {eff }}^{(d)}= & \frac{1}{\Lambda}|H|^{2} \bar{\psi}\left(d_{s}+i d_{s 5} \gamma_{5}\right) \psi \\
& +\frac{1}{\Lambda}\left(H^{*} t_{a} H\right) \bar{\psi}\left(d_{t}+i d_{t 5} \gamma_{5}\right) T_{a} \psi,
\end{aligned}
$$

where $t_{a} \equiv \sigma_{a} / 2$ with $\sigma_{a}$ the Pauli matrices, and the coefficients are dimensionless and of $\mathcal{O}(1)$. In what follows, we study the phenomenology of these $\mathrm{SU}(2)_{L}$ multiplets in the presence of the effective operators (2) and (3), and discuss the constraints on $\Lambda$ for each $Y$.

\section{INELASTIC SCATTERING}

As mentioned above, the effective operators in Eq. (2) generate the mass splitting between the neutral components after the electroweak symmetry breaking. Once the Higgs field gets a vacuum expectation value, $\langle H\rangle=(0, v)^{T} / \sqrt{2}$ with $v \simeq 246 \mathrm{GeV}$, the operators yield the mass splitting as

$$
\Delta m=\frac{v^{4 Y} C_{j Y}\left|c_{s}\right|}{2^{(2 Y-1)} \Lambda^{(4 Y-1)}} .
$$

Here we define $C_{j Y} \equiv\langle j Y j Y \mid(2 Y)(2 Y)\rangle$.

If $\Delta m<\mathcal{O}(100) \mathrm{keV}$, the inelastic scattering of the DM with a nucleus may occur via the $Z$-boson exchange processes, which is significantly restricted by the direct detection experiments. The scattering cross section is

$$
\sigma_{\text {inel }}=\frac{G_{F}^{2} Y^{2}}{2 \pi}\left[N-\left(1-4 \sin ^{2} \theta_{W}\right) Z\right]^{2} M_{\text {red }}^{2} .
$$

Here, $G_{F}$ is the Fermi constant, $\theta_{W}$ is the weak mixing angle, $M_{\text {red }}$ is the reduced mass in the DM-target nucleus system, and $Z$ and $N$ are the numbers of protons and neutrons in the nucleus, respectively. By using the cross section, we obtain the differential event rate with the recoil energy $E_{R}$ in a direct detection experiment as

$$
\frac{d R}{d E_{R}}=\frac{N_{T} m_{T} \rho_{\mathrm{DM}}}{2 m_{\mathrm{DM}} M_{\mathrm{red}}^{2}} \sigma_{\text {inel }} F^{2}\left(E_{R}\right) \int_{v_{\min }}^{\infty} \frac{f(v)}{v} d v,
$$

where $N_{T}$ is the number of the target nuclei, $m_{\mathrm{DM}}$ and $m_{T}$ are the masses of the DM and the nucleus, respectively, $\rho_{\mathrm{DM}}$ is the local DM density, $f(v)$ is the local DM velocity distribution, and $F^{2}\left(E_{R}\right)$ denotes a nuclear form factor. The minimum speed $v_{\min }$ in the integral is given by

\footnotetext{
${ }^{3}$ Dipole-type operators are usually suppressed by a loop factor, and thus their contribution is subdominant.

${ }^{4}$ For recent related studies, see Refs. [7,8].
} 


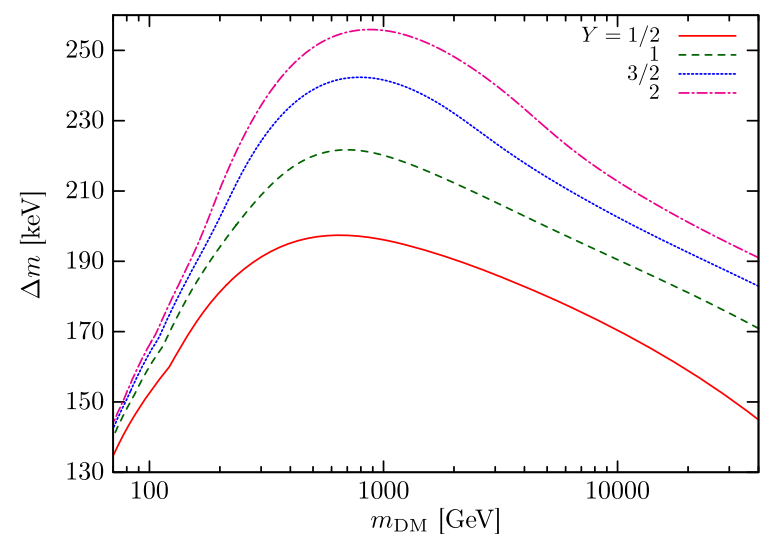

FIG. 1 (color online). Lower bound on the mass splitting $\Delta m$ from the inelastic-scattering limits as functions of the DM mass $m_{\mathrm{DM}}$.

$$
v_{\min }=\frac{c}{\sqrt{2 m_{T} E_{R}}}\left(\frac{m_{T} E_{R}}{M_{\mathrm{red}}}+\Delta m\right) .
$$

Current direct detection experiments have sensitivities to a recoil energy of $E_{R}<\mathcal{O}(100) \mathrm{keV}$, and thus the event rate $R$ strongly depends on $\Delta m$ if $\Delta m<\mathcal{O}(100) \mathrm{keV}$, while the scattering basically never happens if $\Delta m \gg 1 \mathrm{meV}$. As a consequence, the direct detection experiments impose a lower limit on the mass difference, which is interpreted as an upper limit on the scale $\Lambda$ through the relation in Eq. (4).

In Fig. 1, we show the constraints on the mass splitting $\Delta m$ coming from the direct detection experiments as functions of the DM mass $m_{\mathrm{DM}}$. The $Y=1 / 2,1,3 / 2,2$ cases are presented from bottom to top. We combine the results of XENON10 [9], XENON100 [10], and LUX [11], and give the lower limits at $90 \%$ C.L. by using a simple merging and maximum gap method [12,13]. We use the same parameters for the nuclear form factor and the astrophysical DM velocity distribution as Ref. [9], except for $v_{\text {esc }}=544 \mathrm{~km} / \mathrm{s}$ [14]. Although the constraints strongly depend on these parameters, the limit $\Delta m>$ $100 \mathrm{keV}$ is robust in a range of the DM masses shown in the figure. As a result, we have the upper bounds on $\Lambda$ as

$\Lambda \lesssim\left(10^{9}, 3 \times 10^{4}, 4 \times 10^{3}\right) \mathrm{GeV}$ for $Y=\left(\frac{1}{2}, 1, \frac{3}{2}\right)$

A larger $Y$ leads to a smaller $\Lambda$, which gives significant impacts on low-energy observables, as we will see below.

\section{ELASTIC SCATTERING}

As we have discussed so far, by considering the inelasticscattering processes, we obtain an upper limit on the scale $\Lambda$ for each multiplet. If the scale is low enough, on the other hand, the dimension-five operators in Eq. (3) get significant. The operators induce the DM-nucleon elastic scatterings, which are again constrained by the direct detection experiments. Let us evaluate the cross sections. In the presence of the effective operators, the DM-quark scalar coupling $f_{q}$ is induced as

$$
f_{q}=-\frac{1}{2 m_{h}^{2} \Lambda}\left(d_{s}+\frac{Y}{2} d_{t}\right)
$$

where $m_{h}$ denotes the mass of the Higgs boson. Here we neglect the contribution of the operators in Eq. (2). It is actually subdominant when $Y \geq 1$. In the following analysis we use Eq. (9) for the $Y=1 / 2$ case as well, for brevity. The inclusion of the contribution is straightforward; see Ref. [8] for details.

The DM-quark scalar coupling induces the effective coupling of the DM with nucleons. The DM-proton coupling is, for instance, given by

$$
\frac{f_{p}}{m_{p}}=\sum_{q=u, d, s} f_{q} f_{T_{q}}+\frac{2}{27} \sum_{Q=c, b, t} f_{Q} f_{T_{G}} .
$$

Here, $m_{p}$ is the proton mass, and $f_{T_{u}}=0.019$, $f_{T_{d}}=0.027, f_{T_{s}}=0.009$, and $f_{T_{G}} \equiv 1-\sum_{q=u, d, s} f_{T_{q}}$. They are extracted from the recent results of the lattice QCD simulations [15]. In addition, electroweak gauge boson loop diagrams contribute to the effective coupling. The contribution is computed as [16]

$$
f_{p}^{\mathrm{EW}}=\left(n^{2}-1-4 Y^{2}\right) f_{p}^{W}+Y^{2} f_{p}^{Z}
$$

with $f_{p}^{W} \simeq 2.3 \times 10^{-11} \mathrm{GeV}^{-2}$ and $f_{p}^{Z} \simeq-1.1 \times 10^{-10} \mathrm{GeV}^{-2}$. These values scarcely depend on the DM mass when it is larger than the gauge boson masses. The spin-independent (SI) DM-proton elastic scattering cross section $\sigma_{\mathrm{SI}}^{p}$ is then given by

$$
\sigma_{\mathrm{SI}}^{p}=\frac{4}{\pi} M_{\mathrm{red}}^{2} f_{p}^{2}
$$

In Fig. 2, we show the DM-proton SI scattering cross sections $\sigma_{\mathrm{SI}}^{p}$ as functions of $\Lambda$ for some selected model parameters. The $Y=j=1 / 2,1,3 / 2,2$ cases are plotted from bottom to top. We set $m_{\mathrm{DM}}=1 \mathrm{TeV}, d_{s}=1$, and the other coefficients to be zero. The cross sections turn out to be almost independent of the DM mass. The blue shaded region represents the current experimental bound given by the LUX Collaboration [11]. As can be seen, it has already restricted the region of $\Lambda<\mathrm{a}$ few TeV for $m_{\mathrm{DM}}=1 \mathrm{TeV}$. We also show the expected sensitivities of the future Xenon-based experiments [17] in black dashed lines for reference. It is found that the future experiments can probe $\Lambda=\mathcal{O}\left(10^{(4-5)}\right) \mathrm{GeV}$, which is significantly higher than the conditions from the inelastic-scattering limits (8) for $Y \geq 1$. In addition, larger $Y$ and $n$ tend to yield larger scattering rates via the electroweak loop contributions, which are independent of $\Lambda$. Anyway, we see that larger $Y$ 


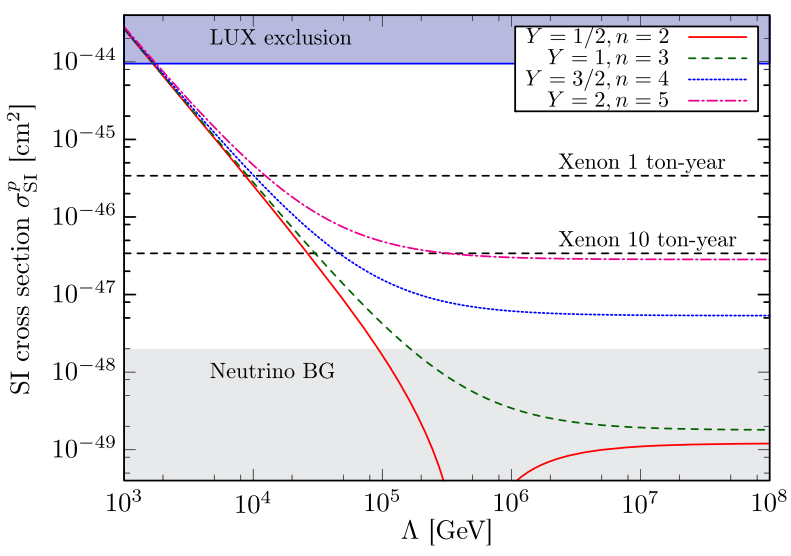

FIG. 2 (color online). DM-proton SI scattering cross sections as functions of $\Lambda$. We set $m_{\mathrm{DM}}=1 \mathrm{TeV}, d_{s}=1$, and the others' coefficients to be zero. Blue shaded region represents the present bound given by the LUX experiment [11], while gray shaded region indicates the border line below which the neutrino background dominates the DM signals [18].

scenarios can be relatively easily tested with future DM detection experiments.

\section{ELECTRIC DIPOLE MOMENTS}

The DM direct detection bounds discussed above are relevant to the parity-even part of the effective operators. The parity-odd part is, on the other hand, probed or constrained with the EDMs. The experimental constraints on the quantities give another lower limit on the scale $\Lambda$. The EDM of a fermion $f$ is induced at the two-loop level ${ }^{5}$ through the so-called Barr-Zee diagrams [19], which is computed as follows:

$$
d_{f}=d_{f}^{h \gamma}+d_{f}^{h Z}+d_{f}^{W W},
$$

with

$$
\begin{aligned}
d_{f}^{h \gamma}= & \frac{e^{3} Q_{f} m_{f} n}{3(4 \pi)^{4} \Lambda \mu} f_{0}\left(\frac{\mu^{2}}{m_{h}^{2}}\right) \\
& \times\left[\left(n^{2}-1+12 Y^{2}\right) d_{s 5}-Y\left(n^{2}-1\right) d_{t 5}\right], \\
d_{f}^{h Z}= & \frac{e g^{2} m_{f} n}{12(4 \pi)^{4} \Lambda \mu}\left(T_{f}^{3}-2 Q_{f} \sin ^{2} \theta_{W}\right) f_{1}\left(\frac{m_{Z}^{2}}{m_{h}^{2}}, \frac{\mu^{2}}{m_{h}^{2}}\right) \\
\times & {\left[2\left\{\left(n^{2}-1\right)-12 Y^{2} \tan ^{2} \theta_{W}\right\} d_{s 5}\right.} \\
- & \left.Y\left(n^{2}-1\right)\left(1-\tan ^{2} \theta_{W}\right) d_{t 5}\right], \\
& d_{f}^{W W}=\frac{e g^{2} m_{f} T_{f}^{3}}{6(4 \pi)^{4} \Lambda \mu} Y n\left(n^{2}-1\right) d_{t 5} f_{0}\left(\frac{\mu^{2}}{m_{W}^{2}}\right) .
\end{aligned}
$$

${ }^{5} \mathrm{EDM}$ are, in general, induced also at one-loop level through the effects of UV physics above the scale $\Lambda$. As long as $|\mu| \ll \Lambda$ holds, however, such a contribution is subdominant.
Here, $e=|e|$ is the positron charge, $g$ is the $\mathrm{SU}(2)_{L}$ coupling constant, and $m_{f}, Q_{f}$, and $T_{f}^{3}$ are the mass, electric charge in the unit of $e$, and isospin of the fermion $f$, respectively. The mass functions in the expressions are

$$
\begin{aligned}
& f_{0}(r)=r \int_{0}^{1} d x \frac{1}{r-x(1-x)} \ln \left(\frac{r}{x(1-x)}\right), \\
& f_{1}\left(r_{1}, r_{2}\right)=\frac{1}{1-r_{1}}\left[f_{0}\left(r_{2}\right)-r_{1} f_{0}\left(\frac{r_{2}}{r_{1}}\right)\right] .
\end{aligned}
$$

Currently the electron EDM bound $\left|d_{e}\right|<8.7 \times 10^{-29} e \mathrm{~cm}$ by the ACME Collaboration [20] gives the most stringent limit on the UV-physics scale. For the electron EDM, the $h \gamma$ and $W W$ contributions are dominant. The prefactor of Eq. (14) is

$\frac{e^{3} Q_{e} m_{e}}{3(4 \pi)^{4} \Lambda \mu} f_{0} \simeq-3 \times 10^{-29} e \mathrm{~cm} \times\left(\frac{10^{6} \mathrm{GeV}^{2}}{\Lambda \mu}\right) \ln \left|\frac{\mu}{m_{h}}\right|$.

With $\mathcal{O}(1) C P$-violating coefficients $d_{s 5}$ and $d_{t 5}, \Lambda$ less than several $\mathrm{TeV}$ is disfavored for $m_{\mathrm{DM}}=\mathcal{O}(1) \mathrm{TeV}$.

The sensitivity of the EDM measurements is expected to be improved by a few orders of magnitude in the future $[21,22]$; e.g., $\left|d_{e}\right| \sim 10^{-31} e \mathrm{~cm}$. With the improved measurements the cutoff scale $\Lambda$ even above the $\mathrm{PeV}$ scale can be tested.

\section{SUMMARY AND DISCUSSION}

We have studied the electroweak interacting DM with nonzero hypercharge. With the higher-dimensional operators (2), dangerous $Z$-boson mediated scatterings can be avoided if they give the mass splitting $\Delta m \gtrsim 100 \mathrm{keV}$ between the neutral components. However, other operators with the same cutoff scale $\Lambda$ may induce large signals for the DM-nucleus elastic scatterings and/or the EDMs. In Fig. 3, we show the complementary feature for some selected examples. Here, we take $d_{s}=d_{s 5}=c_{s}=1$ and $d_{t}=d_{t 5}=0$. Each hatched region filled with (without) the same color shows the current constraints (prospects). For prospects we refer to the expected reach of a Xenon-based 10 ton-year experiment [17] for the direct detection limits and $\left|d_{e}\right|=10^{-31} \mathrm{ecm}$ for the EDM bounds [21,22]. Generally speaking, a larger $n$ with $Y$ fixed leads to a more severe limit. Note that when the cutoff scale $\Lambda$ approaches the DM mass, analyses based on the effective theories become invalid. Constraints in such a case should be dependent on each UV model, since it implies an additional sector showing up around the $\mathrm{TeV}$ scale. Generically, however, we may expect more direct effects on the EDM and DM signals, as well as on the data in the indirect DM searches and the collider experiments, coming from this sector. The contributions make the DM more restricted. Keeping this notice in mind, in Fig. 3 we extrapolate the results computed in effective theories, just 
ELECTROWEAKLY-INTERACTING DIRAC DARK MATTER

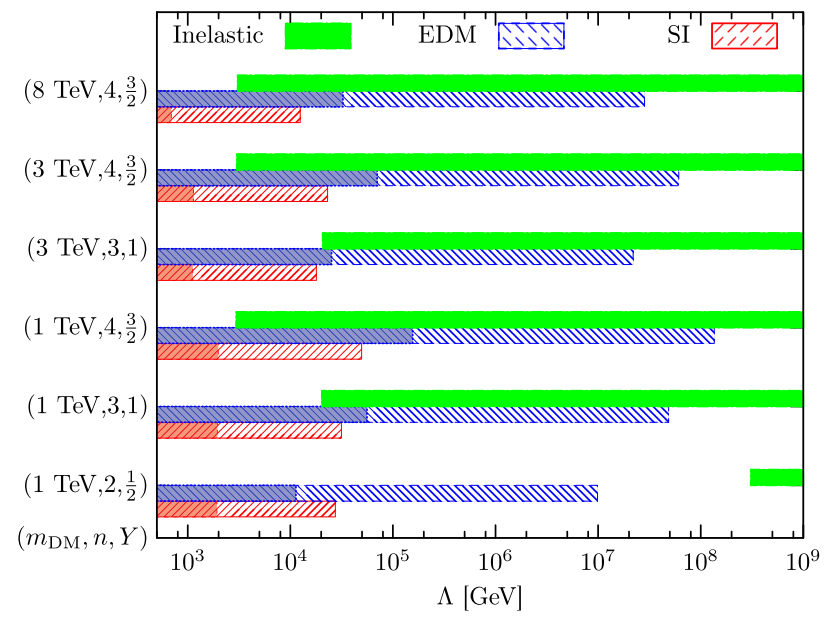

FIG. 3 (color online). Constraints and prospects for the cutoff scale $\Lambda$. We set $d_{s}=d_{s 5}=c_{s}=1$ and $d_{t}=d_{t 5}=0$. Each hatched region filled with (without) the same color shows the current constraints (prospects).

for references. From this figure, it is found that the DM with $Y \geq 3 / 2$ are now strongly disfavored. Even the $Y=1$ cases start to be constrained, and future experiments can examine the cases. If no signal is observed, only the $Y=0$ and $1 / 2$ cases may be the remaining possibilities for the $\mathrm{SU}(2)_{L}$ charged fermionic DM candidates.
PHYSICAL REVIEW D 91, 055035 (2015)

Finally, we briefly comment on the scalar DM cases. Similarly to the Dirac fermion DM, a scalar DM with nonzero hypercharge also has the vector coupling to $Z$-boson. For the $Y \geq 1$ cases, only nonrenormalizable operators can induce the mass splitting between the neutral components to avoid the coupling. Thus, in the case of the $Y \geq 1$ scalar DM, the inelastic bound can give an upper limit on the UV-physics scale, just like the fermion DM cases. We have $\Lambda \lesssim\left(10^{5}, 4 \times 10^{3}\right) \mathrm{GeV}$ for $Y=1,3 / 2$, respectively, with $m_{\mathrm{DM}}=1 \mathrm{TeV}$. On the other hand, the DM-nucleus elastic scattering via the Higgs-boson exchange is induced by renormalizable interactions, and thus it is not necessarily dependent on the UV scale. Further, EDMs are not induced and thus play no role in the scalar DM cases. Nonetheless, when an upper limit on $\Lambda$ is as low as the DM mass, it indicates the presence of extra particles other than the DM multiplet around the $\mathrm{TeV}$ scale, which provides us various ways to probe the scalar DM in experiments.

\section{ACKNOWLEDGMENTS}

The work of N. N. is supported by Research Fellowships of the Japan Society for the Promotion of Science for Young Scientists.
[1] P. Ade et al. (Planck Collaboration), Astron. Astrophys. 571, A16 (2014).

[2] R. Essig, Phys. Rev. D 78, 015004 (2008).

[3] M. Cirelli, N. Fornengo, and A. Strumia, Nucl. Phys. B753, 178 (2006); M. Cirelli, A. Strumia, and M. Tamburini, Nucl. Phys. B787, 152 (2007); M. Cirelli and A. Strumia, New J. Phys. 11, 105005 (2009).

[4] M. Kadastik, K. Kannike, and M. Raidal, Phys. Rev. D 81, 015002 (2010); M. Kadastik, K. Kannike, and M. Raidal, Phys. Rev. D 80, 085020 (2009); M. Frigerio and T. Hambye, Phys. Rev. D 81, 075002 (2010).

[5] E. J. Chun, J.-C. Park, and S. Scopel, J. Cosmol. Astropart. Phys. 12 (2012) 022; Z. Kang, Y. Liu, and G.-Z. Ning, J. High Energy Phys. 09 (2013) 091.

[6] B. Feldstein, M. Ibe, and T. T. Yanagida, Phys. Rev. Lett. 112, 101301 (2014).

[7] J. Hisano, D. Kobayashi, N. Mori, and E. Senaha, Phys. Lett. B 742, 80 (2015).

[8] N. Nagata and S. Shirai, J. High Energy Phys. 01 (2015) 029.

[9] J. Angle et al. (XENON10 Collaboration), Phys. Rev. D 80, 115005 (2009).

[10] E. Aprile et al. (XENON100 Collaboration), Phys. Rev. Lett. 109, 181301 (2012).

[11] D. Akerib et al. (LUX Collaboration), Phys. Rev. Lett. 112, 091303 (2014).
[12] S. Yellin, Phys. Rev. D 66, 032005 (2002).

[13] S. Yellin, arXiv:1105.2928.

[14] M. C. Smith, G. Ruchti, A. Helmi, R. Wyse, J. Fulbright et al., Mon. Not. R. Astron. Soc. 379, 755 (2007).

[15] R. Young and A. Thomas, Phys. Rev. D 81, 014503 (2010); H. Ohki, K. Takeda, S. Aoki, S. Hashimoto, T. Kaneko, H. Matsufuru, J. Noaki, and T. Onogi (JLQCD Collaboration), Phys. Rev. D 87, 034509 (2013).

[16] J. Hisano, K. Ishiwata, N. Nagata, and T. Takesako, J. High Energy Phys. 07 (2011) 005; J. Hisano, K. Ishiwata, and N. Nagata, Phys. Lett. B 690, 311 (2010); J. Hisano, K. Ishiwata, and N. Nagata, Phys. Rev. D 82, 115007 (2010).

[17] K. Arisaka, C. Lam, P. Smith, P. Beltrame, C. Ghag et al., Astropart. Phys. 36, 93 (2012).

[18] J. Billard, L. Strigari, and E. Figueroa-Feliciano, Phys. Rev. D 89, 023524 (2014).

[19] S. M. Barr and A. Zee, Phys. Rev. Lett. 65, 21 (1990).

[20] J. Baron et al. (ACME Collaboration), Science 343, 269 (2014).

[21] J. J. Hudson, D. M. Kara, I. J. Smallman, B. E. Sauer, M. R. Tarbutt, and E. A. Hinds, Nature (London) 473, 493 (2011).

[22] A. C. Vutha, W. C. Campbell, Y. V. Gurevich, N. R. Hutzler, M. Parsons et al., J. Phys. B 43, 074007 (2010). 